\title{
Le rôle majeur du canal potassique TREK-1 dans la protection neuronale induite par les oméga-3
}

\author{
Catherine HEURTEAUX \\ Nicolas BLONDEAU \\ Institut de pharmacologie moléculaire \\ et cellulaire, \\ UMR CNRS 6097 \\ 660 Route des Lucioles, \\ 06560 Valbonne, France \\ $<$ heurteau@ipmc.cnrs.fr>
}

\begin{abstract}
The nutritional interest of polyunsaturated fatty acids from omega-3, that are mainly present in vegetal and fish oils is now validated by the scientific community. Their beneficial effects have first been reported in coronary heart diseases. Many neurological and chronic diseases are often related to deficiencies in omega-3 and omega- 6 and their derivatives. Polyunsaturated fatty acids from omega-3 family are essential to brain growth and neuronal preserving (foetuses, children, old people) as well as visual and cognitive functions. They are recently considered as factors of improvement in some mental diseases. Today, polyunsaturated fatty acids could play a key role in the prevention and/or or the treatment of cerebral diseases. With the development of in vitro and in vivo experimental models, it is now possible to demonstrate the omega-3-induced neuronal protection against major pathologies such as epileptic seizures and cerebral ischemia. The molecular mechanism of neuronal protection induced by omega-3 is now clarified. The omega-3 target would be a potassium channel, TREK-1, which belongs to the new family of 2-P domain potassium channels (K-2P). The discovery of the physiopathological role of these $K-2 P$ channels can represent an important therapeutical challenge not only in cerebrovascular diseases, but also in psychiatry.
\end{abstract}

Key words: omega-3, alpha linolenic acid (ALA), 2P-domain $K^{+}$channels (K-2P), TREK-1, epilepsy, cerebral ischemia

Les acides gras polyinsaturés (AGPI), incorporés dans les phospholipides membranaires sont des constituants structuraux fondamentaux du système nerveux central (SNC) dont la teneur conditionne le fonctionnement des cellules neuronales. Ils représentent environ $20 \%$ de la matière sèche cérébrale et sont constitués des familles oméga-3 $(n-3)$ et oméga- $6(n-6)$. Les acides gras précurseurs de ces deux familles (acide alphalinolénique et acide linoléique) sont trouvés en quantités appréciables dans certaines huiles végétales (colza, noix et soja) et sont considérés comme des acides gras essentiels. Ne pouvant pas être synthétisés de novo par l'homme et les animaux, leur apport ne peut se faire que par l'alimentation. L'acide alphalinolénique (ALA, $18: 3 n-3)$, précurseur des longues chaînes oméga-3, peut être transformé par l'homme en dérivés à longues chaînes (LC-n-3), dont les plus importants sont les acides eicosapentaénoïque (EPA, 20:5n-3) et docosahexanoïque (DHA, 22:6n-3), qui peuvent aussi être apportés par la consommation de poissons gras. L'acide linoléique ( $L A, 18: 2 n-6)$, précurseur des oméga-6 est transformé par I'homme en acide arachidonique (AA, 20:4n-6), LC-n-6 que I'on trouve aussi dans les viandes animales terrestres. Un déséquilibre dans le rapport LA/ALA dans l'apport alimentaire (optimal aux alentours de 5) est source de déséquilibres métaboliques et peut avoir de profondes répercussions sur divers processus physiologiques [1].

\section{L'enjeu thérapeutique des AGPI}

La prise alimentaire d'AGPI a de nombreuses conséquences biologiques, en particulier dans les domaines intéressant le risque cardiovasculaire (thrombose, métabolisme lipidique, inflammation). Les premiers effets bénéfiques des AGPI ont été décrits au niveau des maladies coronariennes, par la prévention de la thrombose et de l'athérosclérose associée à un effet anti-arythmique, antiagrégant et hypotriglycéridémiant [2-7]. L'engouement pour leur intérêt nutritionnel ne faisant que croître, les nombreuses études qui en découlent impliquent dorénavant les AGPI dans de nombreux mécanismes physiologiques et comportementaux. Les AGPI sont impliqués dans diverses maladies inflammatoires [8], ainsi que dans le diabète et l'ostéoporose. Le statut en AGPI constitue un des facteurs environnementaux capables d'affecter le fonctionnement du SNC. Des déficits comportementaux et cognitifs (apprentissage, mémorisation et habituation) associés à des anomalies du métabolisme de certains neuromédiateurs apparaissent chez des animaux recevant des régimes déséquilibrés en AGPI. Des taux anormaux d'AGPI sont détectés chez des humains souffrant de maladies neurologiques [9]. Les oméga-3 semblent efficaces dans la prévention du stress, de la dépression et de la démence, notamment au cours de la maladie d'Alzheimer et atténuent les symptômes de la psychose maniaco-dépressive et de la schizophrénie [9-13]. Enfin,
FONDAMENTAL
Reçu le 29/11/04

Accepté le 22/02/05 
les AGPI sont importants dans le développement cérébral, I'acquisition des capacités visuelles et le développement des fonctions cérébrales, notamment motrices et intellectuelles [14].

Des études récentes se sont attachées à démontrer l'effet protecteur des AGPI, et principalement des oméga-3 face aux crises d'épilepsie et à l'ischémie cérébrale [15-17]. Les accidents vasculaires cérébraux (AVC) touchent plus d'un million de personnes chaque année dans I'Union européenne. En France, ils constituent la troisième cause de mortalité après les maladies cardiovasculaires et les affections malignes, avec une incidence annuelle de 125000 nouveaux cas (1,5/100 000/an). Le nombre de personnes atteintes d'AVC est estimé à 500000 . Ils représentent en outre la première cause de handicap d'origine neurologique chez l'adulte. C'est aussi une cause majeure de démence, de dépression et d'épilepsie secondaires à l'ischémie. Les AVC sont des complications aiguës (soudaines) d'une maladie vasculaire, dus à un arrêt de la vascularisation sanguine dans un territoire cérébral (infarctus cérébral) ou à une rupture d'un vaisseau (hémorragie cérébroméningée). Parmi les AVC, $80 \%$ sont d'origine ischémique, contre $20 \%$ en rapport avec une hémorragie. Malgré les efforts en matière de prévention (mesures contre I'hypertension artérielle, le diabète, le tabagisme ou encore les dyslipidémies), qui ont réduit l'incidence de l'AVC de 30 à $50 \%$ selon les études, la prise en charge des patients victimes d'AVC reste pauvre sur le plan thérapeutique. En effet, hormis la thrombolyse intraveineuse, dont les indications n'atteignent que 5 à $10 \%$ des patients, aucune thérapeuti- que n'a rencontré de succès objectif. Une des conséquences fréquentes d'un AVC est le développement d'une épilepsie du lobe temporal (TLE) subséquente. Caractérisée par des convulsions partielles récurrentes, la TLE est une forme d'épilepsie qui affecte plus d' $1 \%$ de la population mondiale et qui est souvent associée à une pharmacorésistance.

Ainsi, la recherche de nouvelles cibles thérapeutiques capables de protéger les neurones d'une ischémie cérébrale et de crises d'épilepsie demeure un challenge important. Notre équipe dans le laboratoire du Professeur M. Lazdunski a montré que la découverte d'une nouvelle classe structurale de canaux potassiques, appelés canaux $\mathrm{K}^{+}$à deux domaines $P(K-2 P)[18,19]$, permet d'envisager une nouvelle stratégie de protection neuronale.

\section{Propriétés moléculaires et fonctionnelles des canaux K-2P}

Exprimés de façon ubiquitaire dans l'organisme, les canaux $\mathrm{K}^{+}$ont un rôle majeur dans les fonctions physiologiques associées à une variation de potentiel électrique membranaire. Ces complexes protéiques permettant la diffusion passive et sélective du $\mathrm{K}^{+}$à travers la membrane plasmique contrôlent dans le système nerveux l'excitabilité neuronale, la transmission synaptique et la libération de certains neurotransmetteurs [20]. Les avancées récentes dans le domaine de la génétique ont permis d'associer chez l'homme les canaux $\mathrm{K}^{+}$à de nombreuses maladies génétiques [21].

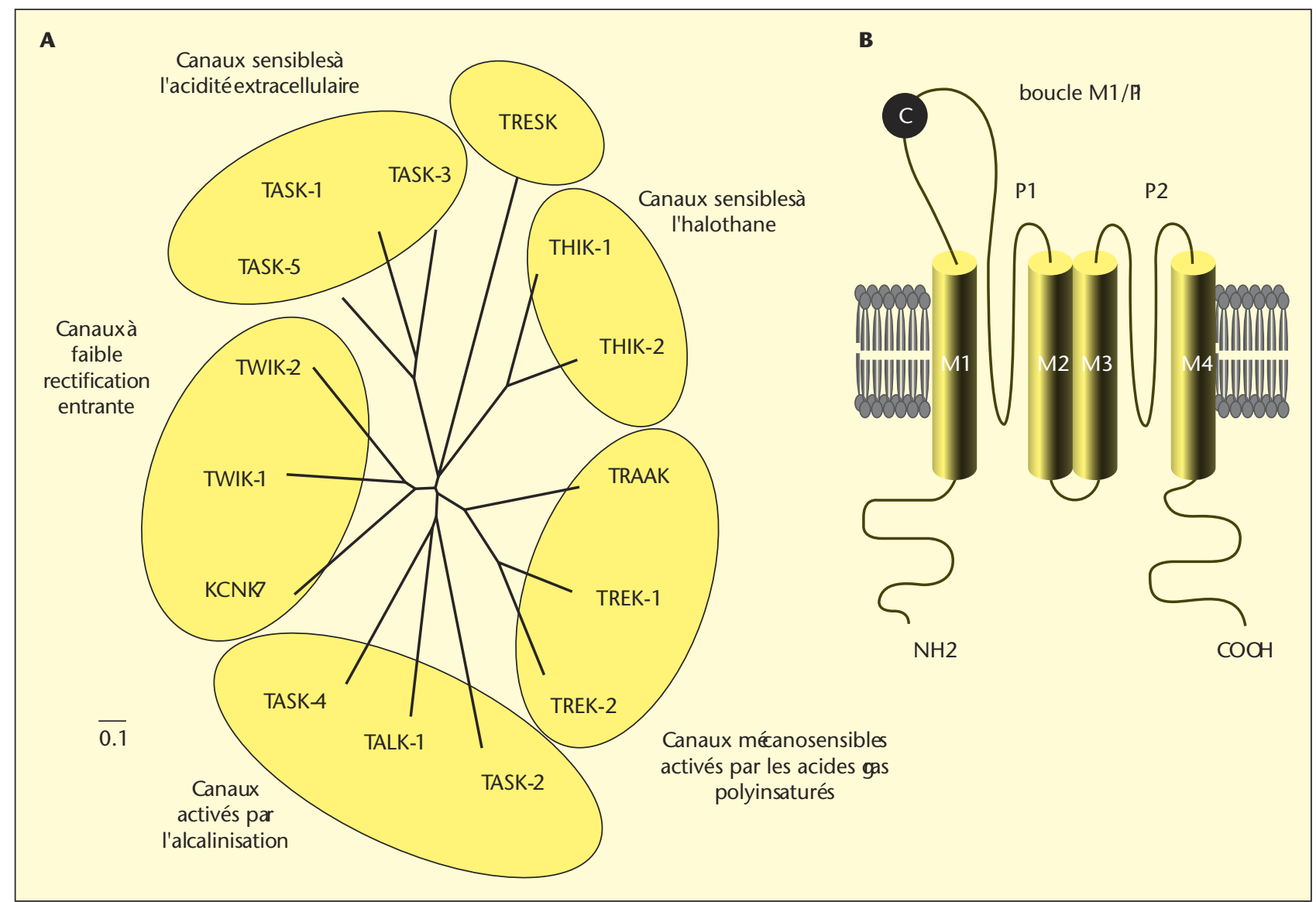

Figure 1. La famille des canaux potassiques à deux domaines P de mammifères (K-2P). A : Arbre phylogénique des différentes sous-unités potassiques K-2P. Les canaux K-2P sont regroupés en 6 sous-groupes selon leurs homologies de séquence et leurs propriétés fonctionnelles : les canaux à faible rectification entrante (TWIK1, TWIK2 et KCNK7); les canaux sensibles à l'acidification extracellulaire (TASK1, TASK3 et TASK5); les canaux activés par I'alcalinisation du milieu extracellulaire (TASK2, TALK1 et TALK2); les canaux mécanosensibles activés par les lipides (TREK1, TREK2 et TRAAK) ; les canaux inhibés par I'halothane (THIK1 et THIK2) et le canal TRESK, dont les propriétés restent encore à définir. B : Structure d'une sous-unité potassique K-2Pà 4 segments transmembranaires et 2 domaines $P$ ( $P$ pour pore). 
Parmi les plus importantes, on peut citer le syndrome du QT long (désordre du système électrique du cœur, avec un allongement de l'intervalle QT favorisant les arythmies ventriculaires, appelées torsades de pointes) et la fibrillation auriculaire au niveau du système cardiaque, la paralysie périodique au niveau musculaire ; le syndrome de Bartter au niveau rénal et l'hyperinsulinémie hypoglycémiante infantile au niveau pancréatique; enfin les épilepsies infantiles et l'ataxie épisodique au niveau du système nerveux et les surdités au niveau sensoriel. Plus de 81 gènes codant pour des sous-unités des canaux $\mathrm{K}^{+}$ont été identifiés chez les mammifères, dont la majorité est exprimée dans le système nerveux. De par leurs propriétés structurales et fonctionnelles particulières, les canaux K-2P ont retenu toute l'attention et font l'objet d'intenses investigations quant à leur(s) rôle(s) physiologique(s).

Les canaux K-2P présentent la caractéristique unique de posséder deux domaines $P$ ( $P$ pour pore) en tandem ( $P 1$ et $P 2$ ) avec 4 segments transmembranaires (M1 à M4) et une large boucle extracellulaire M1/P1 (figure 1B). Les domaines $P$, séquences conservées de 21 acides aminés, participent à la formation du filtre de sélectivité pour l'ion $\mathrm{K}^{+}$. Depuis le clonage du premier membre de cette famille, TWIK1 (pour tandem of $P$ domains in a weak inweardly-rectifying $K^{+}$channel) en 1996, 15 sousunités K-2P ont été identifiées chez les mammifères et classées en 6 sous-groupes selon leurs homologies structurales et fonctionnelles (figure
$1 A)$. Les canaux $\mathrm{K}-2 \mathrm{P}$ fonctionnels sont des homodimères qui peuvent aussi former des hétérodimères. Ils sont présents dans tous les tissus de l'organisme ; cependant, chaque canal a un profil d'expression unique $[18,19]$.

Les canaux K-2P sont largement exprimés dans les systèmes nerveux et périphérique (figure 2B). Sont essentiellement exprimés dans le cerveau, les canaux TWIK-1, TREK-1, TREK-2 (TWIK1-related $K^{+}$channel), TRAAK (TWIK1-related arachidonic acid-stimulated $K^{+}$channel), TASK-1 et TASK-3 (TWIK1-related acid-sensitive channel). Ces canaux produisent des courants instantanés, indépendants du potentiel qui sont qualifiés de courants $\mathrm{K}^{+}$de fond, impliqués dans le contrôle du potentiel de repos membranaire (figure $2 \mathrm{C}$ ). Leur régulation par des agents neuroprotecteurs, les anesthésiques volatils, certains neurotransmetteurs et par une large variété de stimuli physiques et chimiques $[18,19,22]$ a suggéré qu'ils peuvent constituer des cibles intéressantes pour le développement de nouveaux agents pharmacologiques.

En relation avec des pathologies neurologiques, comme l'ischémie cérébrale et l'épilepsie, notre équipe s'est principalement intéressée aux canaux TREK et TRAAK avec l'objectif d'étudier leur rôle dans la protection neuronale. Les canaux TREK sont sensibles aux variations du volume cellulaire. Ils sont ouverts par un étirement de la membrane cellulaire et sont inhibés par une hyperosmolarité extracellulaire induisant une dimi-

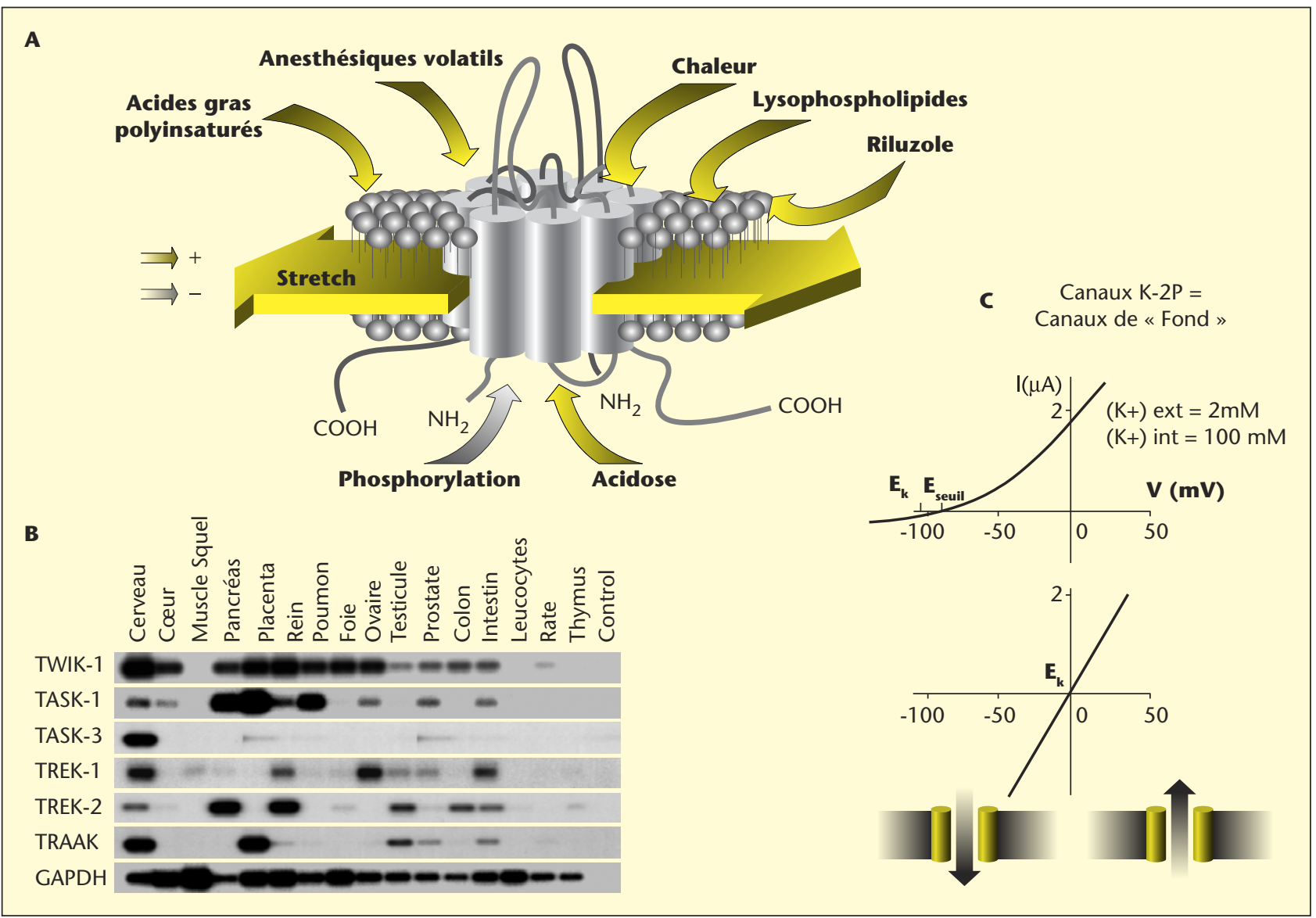

Figure 2. Propriétés fonctionnelles des canaux K-2P. A : Activation polymodale du canal TREK-1 par l'étirement membranaire, les AGPI (dont les oméga-3), le riluzole, les Iysophospholipides et les anesthésiques volatils, la chaleur et l'acidose intracellulaire. TREK-1 est inhibé par la phosphorylation induites par les protéines-kinases A et C (PKA et PKC). B : Distribution tissulaire des canaux K-2P chez la souris adulte. L'expression différentielle des canaux K-2P est obtenue par RT-PCR et Southern Blot utilisant des primers spécifiques comme sondes. GADPH (glycéraldéhyde-3-phosphate déshydrogénase) est utilisé comme contrôle interne de l'intégrité et de la quantité des ADNc. C : Représentation schématique de la courbe courant/potentiel des courants TREK-1. Ces canaux K-2P sont appelés communément canaux de fond (background channel). Ils permettent une sortie continue d'ions $K^{+}$de la cellule et de maintenir le potentiel de repos $\left(E_{k}\right)$ à une valeur négative. Ils sont caractérisés par une courbe courant/potentiel rectifiante sortante pour des concentrations en $\left[\mathrm{K}^{+}\right]$physiologiques. La rectification sortante disparaît à $\left[\mathrm{K}^{+}\right]$symétrique et la courbe courant/potentiel devient linéaire. 
nution du volume cellulaire. Les canaux TREK-1 et TREK-2, mais pas le canal TRAAK, sont activés lors d'une acidose intracellulaire ou lors d'une augmentation de température (figure 2A). Ils sont modulés par les neurotransmetteurs via les récepteurs aux protéines $G[18,19,22]$. Mais la propriété la plus intéressante, quant à la protection neuronale, concerne l'ouverture des canaux TREK et TRAAK par les lysophospholipides à large tête polaire (lysophosphatidylcholine, lysophosphatidylinositol) $[23,24]$ (figure 3C) et le riluzole, utilisé en clinique chez des patients atteints de sclérose latérale amyotrophique [25] (figure 3A). Enfin, les canaux TREK et TRAAK sont activés par les AGPI [24, 26, 27] (figure 3B). Des enregistrements électrophysiologiques dans des cellules transfectées montrent la très forte activation de courants TREK ou TRAAK par l'acide alphalinolénique (ALA, $18: 3 n-3)$ et ses dérivés à longues chaînes, l'acide éicosapentaénoïque (EPA, $20: 3 n$-3) et l'acide docosahexaénoïque (DHA, 22 :6n-3). Comparativement, I'amplitude des courants TREK et TRAAK induits par les acides gras de la famille $n-6$ comme l'acide linoléique (LA, $18: 2 n-6)$ et de son dérivé l'acide arachidonique (AA, $20: 4 n-6)$ est plus faible. Les acides gras saturés comme le palmitate (C16, PAL), le stéarate (C18) et l'arachidate (C20) n'ont quant à eux aucun effet (figure 3B).

\section{Protection neuronale par les AGPI : traitement aigu et prophylactique}

Des modèles expérimentaux permettant de reproduire plusieurs situations pathologiques sous-tendues par une mort neuronale (ischémie cérébrale, crises épileptiques, ischémie de la moelle épinière, glaucome...) ont été développés pour étudier l'effet et les mécanismes $d^{\prime}$ action cellulaires et moléculaires d'agents pharmacologiques potentiellement protecteurs. C'est dans ce contexte que notre équipe a testé les AGPI. Dans un modèle in vivo d'ischémie globale transitoire (10 à 30 min) mimant un infarctus, l'injection intraveineuse d'ALA, à une concentration de $100 \mathrm{nmoles} / \mathrm{kg}$, de 30 à 120 minutes après l'ischémie, protège les neurones pyramidaux de la région CA1 de l'hippocampe, structure la plus vulnérable à l'ischémie. La même injection avec du PAL n'a aucun effet protecteur [16] (figure 4). De même, I'ALA, mais pas le PAL, empêche le développement des crises épileptiques induites par un analogue du glutamate, l'acide kaïnique et protège remarquablement bien les neurones, les $C A 1$ et $C A 3$, sensibles à l'épilepsie, de la mort neuronale [16] (figure $5 A-B$ ). Les oméga-3 protègent aussi de la mort neuronale et de la paralysie après une ischémie transitoire de la moelle épinière [28]. Une alimentation enrichie en DHA induit aussi une résistance des neurones cholinergiques du cerveau de jeunes rats à une lésion cérébrale et prévient la neurodégénérescence [29]. Les mêmes effets bénéfiques des oméga-3 sont aussi observés dans un modèle in vitro d'excitotoxicité sur des neurones glutamatergiques de cervelet en culture [16]. Les AGPI, à I'inverse des acides gras saturés, empêchent l'entrée massive de calcium dans les cellules granulaires et la mort neuronale subséquente, en inhibant la transmission synaptique glutamatergique. La figure 5 C-D montre que l'application d'ALA, DHA ou AA réduit fortement la mort neuronale dans ce modèle in vitro d'excitotoxicité. $D$ 'intenses investigations ont aussi été entreprises dans le cadre de la prévention. Les AGPI induisent une tolérance ischémique et épileptique [15]. Une injection intraveineuse de $500 \mathrm{nmoles} / \mathrm{kg} \mathrm{d}^{\prime} \mathrm{ALA}$, DHA ou AA trois jours avant un épisode ischémique (ischémie globale) ou épileptique (administration d'acide kaïnique) sévère prévient la neurodégénérescence et les crises épileptiques normalement induites par un épisode ischémique ou épileptique sévère (figure $6 A-B$ ). La protection neuronale observée est associée à l'induction de protéines de résistance neuronale anti-apoptotique (type HSP70) et à la prévention de protéines proapoptotiques (type Bax) (figure 6C). Les acides gras saturés comme le PAL n'ont pas d'effet sur la survie neuronale. Cette propriété place les AGPI au rang de molécules capables d'induire un « préconditionnement pharma-

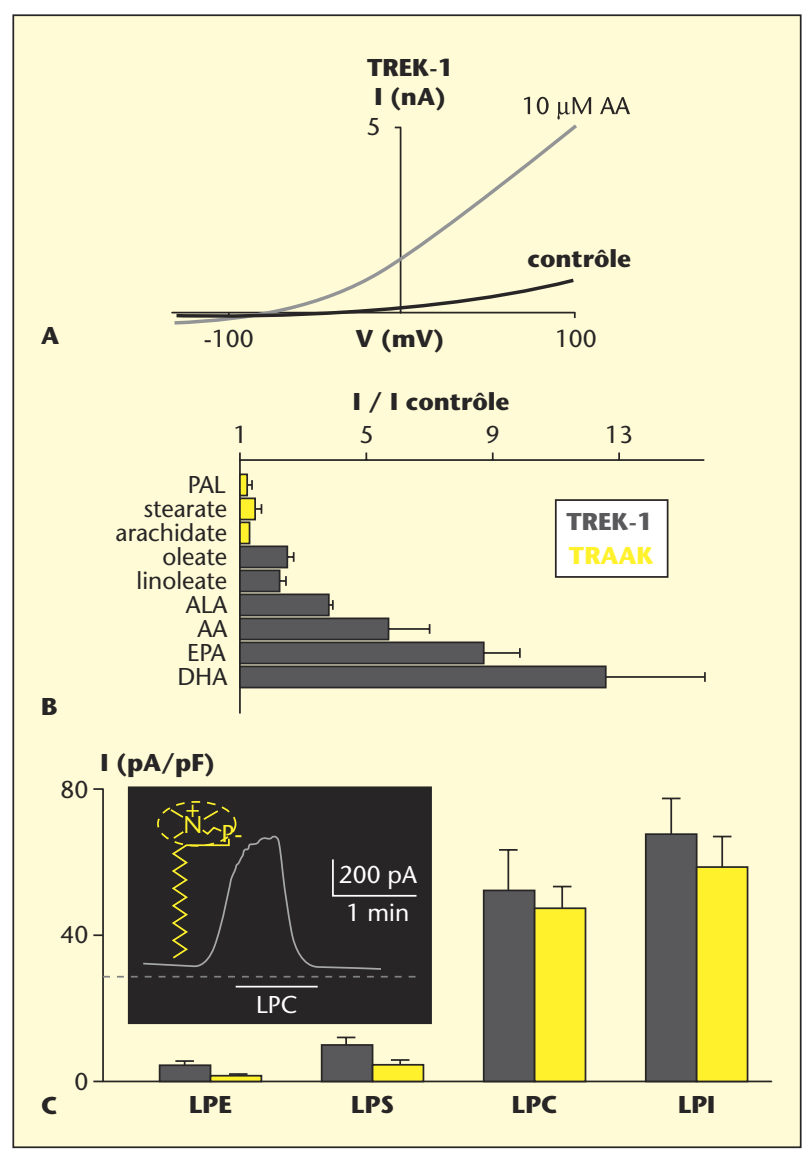

Figure 3. Activation des canaux TREK-1 et TRAAK par les AGPI et les lysophospholipides. $\boldsymbol{A}$ : Effet de $10 \mu \mathrm{M}$ AA sur la courbe I/V (intensité/voltage) en configuration cellule entière d'une cellule COS transfectée avec le canal TREK-1. Les courants TREK-1 sont enregistrés par des rampes de potentiels d'une durée de $600 \mathrm{~ms}$ de -130 à $+100 \mathrm{mV}$. B : Effets des différents AGPI sur l'amplitude du courant TREK-1 dans les cellules COS transfectées. $A A$, acide arachidonique; $A L A$, acide linolénique; $D H A$, acide docosahexanoïque ; EPA, acide eicosapentaénoïque; $L A$, acide linoléique ; PAL, acide palmitique. C : Augmentation de I'amplitude des courants TREK-1 et TRAAK par I'application de $10 \mu \mathrm{M}$ de LPLs en fonction de la taille de leur tête polaire. $\mathrm{PA} / \mathrm{pF}$, picoampères/picofarads. En encadré, activation par $3 \mu \mathrm{M}$ de LPC du courant TREK-1 en configuration cellule entière mesuré à $0 \mathrm{mV}$. LPC, lysophosphatidylcholine; LPE, lysophosphatidyléthanolamine; LPI, lysophosphatidylinositol; LPS, lysophosphatidylsérine.

cologique » en vue d'une stratégie de prévention [15], par exemple dans le cadre d'interventions chirurgicales, et suggère une explication quant aux effets bénéfiques d'une alimentation riche en oméga-3.

\section{Implication du canal TREK-1 dans le mécanisme d'action des oméga-3}

Parce que les AGPI et les lysophospholipides sont de puissants activateurs des canaux TREK et TRAAK, il a été fortement suggéré que ces canaux $\mathrm{K}-2 \mathrm{P}$ pourraient avoir un rôle essentiel dans la résistance neuronale. Au cours d'une ischémie ou d'une crise épileptique, il existe une libération d'AGPI et de lysophospholipides, résultant de la dégradation des phospholipides membranaires par les phospholipases $A_{2}$. Simultanément le $\mathrm{pH}$ intracellulaire chute entraînant une acidose et les neurones gonflent. L'ensemble de ces évènements pourrait contribuer à l'activation des canaux TREK et TRAAK, tant au niveau présynaptique que postsynaptique. De façon intéressante, le riluzole, un important activateur 


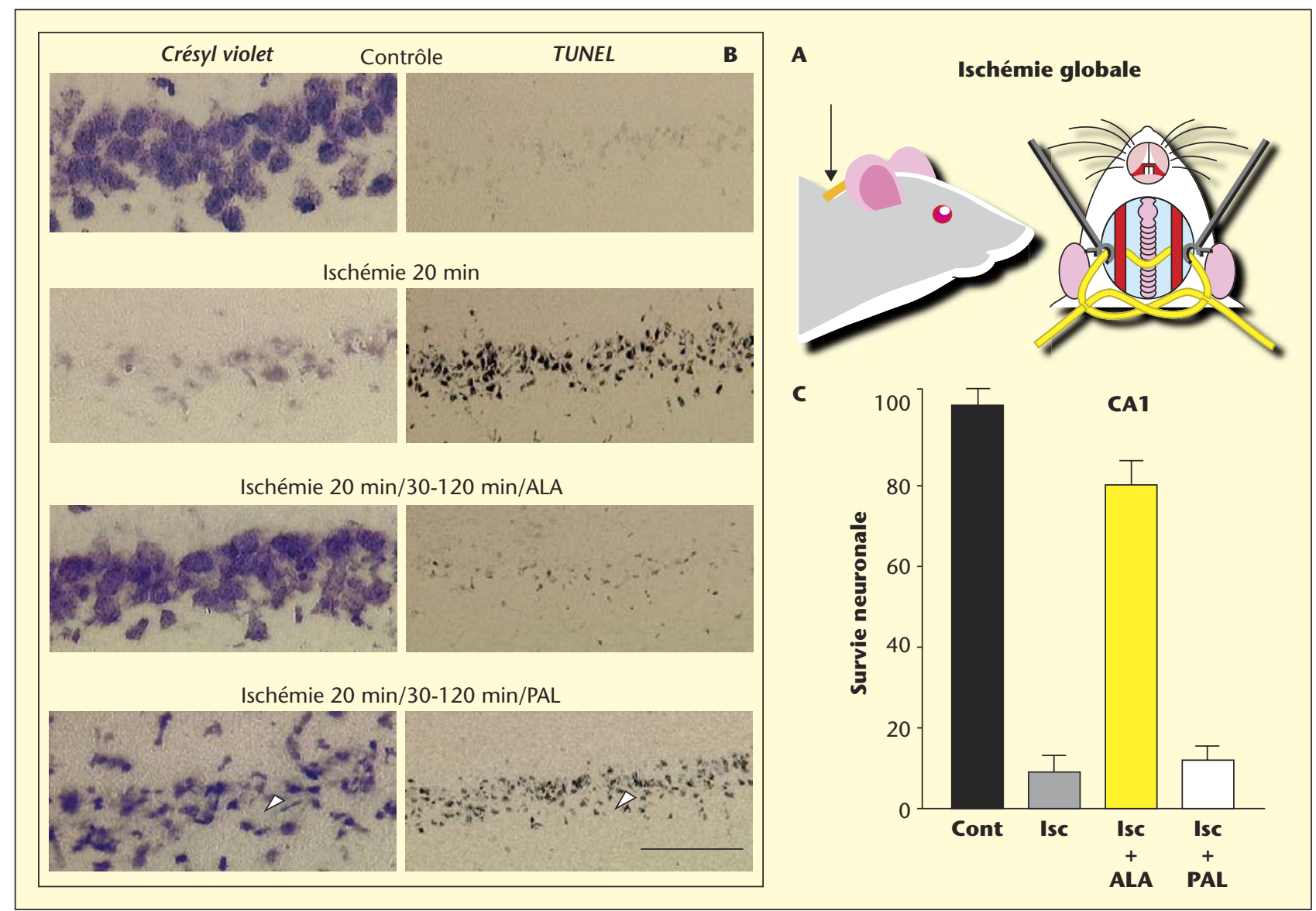

Figure 4. Neuroprotection induite par l'acide linolénique (ALA) dans un modèle d'ischémie globale. $\boldsymbol{A}$ : Illustration du modèle d'ischémie globale chez le rat consistant en la cautérisation des artères vertébrales et le clampage transitoire (20 minutes) des deux carotides. B : Photographies représentatives de la région CA1 de l'hippocampe (la plus vulnérable à l'ischémie) 7 jours (coloration au crésyl violet) ou 3 jours (méthode TUNEL) après l'attaque ischémique. La coloration au crésyl violet permet d'effectuer un comptage des neurones sains et la méthode TUNEL d'identifier les cellules apoptotiques. Les injections d'ALA ou de PAL se font par voie intra-veineuse à une concentration de $100 \mathrm{nmol} / \mathrm{kg}$ dans un délai de 30 à 120 min post-ischémie. C : Histogrammes représentant le pourcentage de survie neuronale 7 jours après l'ischémie.

des canaux TREK et TRAAK, protège aussi de l'ischémie cérébrale et rétinienne $[30,31]$ et se révèle particulièrement efficace dans la prévention de lésions médullaires [32]. D’autres activateurs de ces canaux, comme la température [33], les anesthésiques volatils (halothane, isoflurane, sevoflurane, desflurane...) [34] et les anesthésiques gazeux (xénon, NO et cyclopropane) [35] ont aussi des propriétés neuroprotectrices. Faute de bloqueur spécifique de ces canaux K-2P, la démonstration de leur implication n'était pas aboutie. La production de souris mutantes, dont le gène codant pour le canal TREK-1 a été éliminé (TREK $1^{-/-}$), a récemment permis d'élucider le mécanisme moléculaire de protection contre l'ischémie globale et les crises épileptiques par les oméga-3 [36]. Les souris invalidées pour TREK-1 sont beaucoup plus vulnérables à I'ischémie cérébrale et aux crises épileptiques que les souris normales (figure 7). Cette vulnérabilité des souris invalidées TREK $1^{-/-}$est évaluée par l'intensité des crises épileptiques et par le taux de mortalité induit par I'agression ischémique ou épileptique. Chez les souris TREK $1^{-/-}$soumises à une ischémie globale, on constate une augmentation de $40 \%$ de décès et une susceptibilité à développer des convulsions dans les trois jours suivant l'ischémie, qui ne se produit pas chez les souris TREK $1^{+/+}$. Par ailleurs, les souris TREK $1^{-/}$meurent pour des concentrations d'agents épileptogènes (acide kaïnique, agoniste glutamatergique ou pentylènetétrazole, antagoniste GABAergique) qui laissent les souris sauvages en vie. La découverte la plus importante est la disparition des effets protecteurs des oméga-3 chez les souris TREK1 ${ }^{-/-}$(figure 7). Le même type d'approche sur des souris invalidées pour le canal TRAAK n'a révélé aucune augmentation de la vulnérabilité à l'ischémie ou à l'épilepsie des souris TRAAK ${ }^{-1-}$. Le canal TREK-1 confère donc une résistance à la mort due à l'hyperexcitabilité induite par une ischémie ou une crise épileptique. Ce travail [36] est donc un argument fort pour dire qu'à l'inverse du canal TRAAK, le canal TREK-1 se révèle essentiel dans le contrôle de I'hyperexcitabilité et de l'épileptogenèse et est un facteur clé dans la neuroprotection induite par les oméga-3.

La protéine TREK-1 est très exprimée dans les neurones glutamatergiques et GABAergiques de I'hippocampe, au niveau pré- et post-synaptique $[16,37]$. En termes mécanistiques (figure 8), il est possible d'imaginer que les AGPI et les lysophospholipides libérés au cours de l'épisode ischémique ou épileptique, puis l'acidose et le gonflement cellulaire résultants, puissent ouvrir les canaux TREK-1 présynaptiques. Leur activation induirait une hyperpolarisation des neurones qui entraînerait une diminution de l'activation des canaux $\mathrm{Ca}^{2+}$ sensibles au voltage et consécutivement limiterait l'entrée de calcium et la libération de glutamate dans la fente synaptique. Au niveau post-synaptique, l'hyperpolarisation membranaire diminuerait l'activation du récepteur glutamatergique NMDA (par blocage par le magnésium) et antagoniserait la dépolarisation induite par le récepteur AMPA/kainate. La résultante en serait une diminution de la transmission glutamatergique et de l'excitotoxicité. Toutefois, la stimulation des récepteurs au glutamate métabotropiques (mGlu) couplés aux protéines $\mathrm{Gq}$ devrait tendre à fermer le 


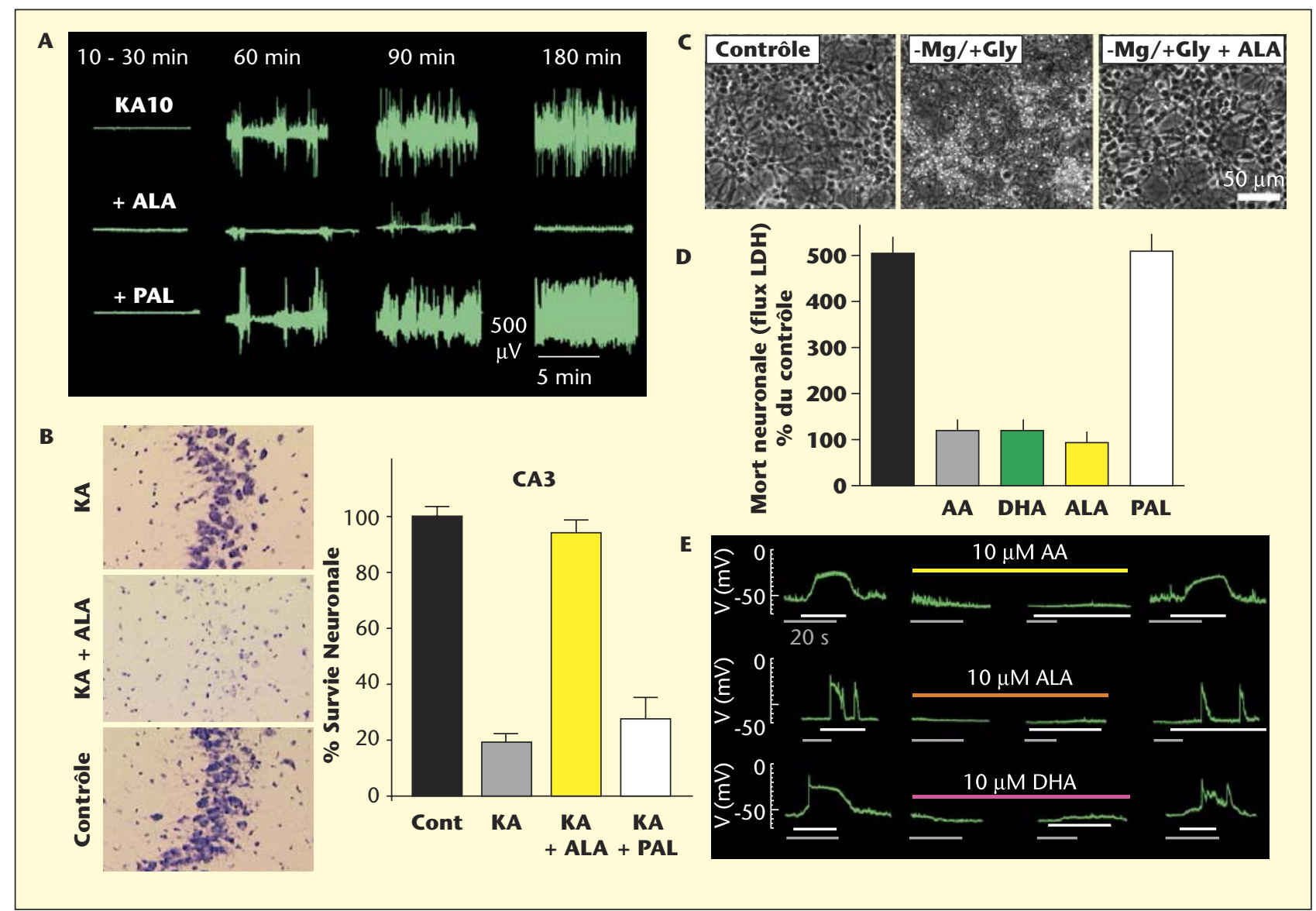

Figure 5. Protection neuronale induite par l'acide linolénique (ALA) dans deux modèles d'excitotoxicité (in vivo et in vitro). $\boldsymbol{A}$ : Enregistrement d'électroencéphalogrammes illustrant les effets d'une injection d'ALA ou de PAL sur les crises épileptiques induites par l'acide kaïnique (10 mg/kg KA). B : Photographies représentatives de la région CA3 de I'hippocampe (la plus vulnérable à l'épilepsie) associées aux histogrammes représentant le pourcentage de survie neuronale 7 jours après I'attaque épileptique. Les injections d'ALA ou de PAL se font par voie intra-veineuse à une concentration de $100 \mathrm{nmol} / \mathrm{kg}$ dans un délai de 30 à 120 min post-épilepsie. C : Effet protecteur de $10 \mu \mathrm{M}$ d'AA contre l'excitotoxicité induite par un milieu-Magnésium/+Glycine dans des cultures granulaires de cervelet. D : Effet de différents AGPI (AA, DHA, ALA et PAL) sur la viabilité cellulaire estimée par le flux de l'enzyme lactate déshydrogénase (LDH). E : Effet protecteur de différents AGPI (AA, ALA, DHA) sur les décharges excitatrices induites par le milieu -Magnésium/+Clycine dans les cellules granulaires de cervelet.

canal TREK-1 [38] et diminuer ainsi la résistance neuronale. Enfin, l'ouverture des canaux TREK1 présynaptiques pourrait aussi provoquer une accumulation de $\mathrm{K}^{+}$dans la fente synaptique, qui devrait être rapidement réabsorbé par les cellules gliales. Le canal TREK-2, présent dans les astrocytes [39] et fortement activé par les AGPI, l'acidose et l'étirement [24] pourrait aussi jouer un rôle important dans l'homéostasie potassique des cellules non excitables du cerveau, et intervenir indirectement dans la neuroprotection.

\section{Conclusion}

Les acides gras polyinsaturés (AGPI) des séries oméga-3 et 6 sont des constituants structuraux essentiels du SNC. L'origine des AGPI est exclusivement alimentaire (graisses animales et végétales). C'est pendant la période périnatale que la qualité des apports en AGPI semble déterminante pour le développement du SNC, notamment pour les processus de myélinisation et de synaptogenèse, et pour la maturation de la fonction visuelle. Or, il se dégage des études épidémiologiques que la consommation en oméga-3 par les populations occidentales est insuffisante, les habitudes alimentaires privilégiant l'apport d'aliments à la fois riches en oméga-6 et pauvres en oméga-3, laissant supposer des conséquences sur le développement neurosensoriel du jeune enfant. Les effets de ces déséquilibres sur les individus dépendent bien évidemment de facteurs constitutionnels et environnementaux propres à chacun, mais il est aujourd'hui admis qu'ils contribuent à l'augmentation de l'incidence de certaines pathologies dans les pays développés, notamment les maladies cardiovasculaires. À la lumière des nombreuses études qui ont vu le jour ces dernières années, on se doit également de prendre en considération ces phénomènes dans l'apparition de certains désordres neuropsychiatriques (dépression, démence et troubles bipolaires), en raison de I'importance fondamentale des AGPI dans le développement et le fonctionnement du SNC. En effet, il est maintenant établi que la présence d'AGPI oméga-3 dans les membranes des cellules nerveuses influence le fonctionnement de certains systèmes de neurotransmission, notamment monoaminergiques et cholinergiques [40]. De nombreuses hypothèses sur les mécanismes d'action spécifiques des acides gras essentiels dans le cerveau ont été avancées, mais sans jamais de démonstration moléculaire fondamentale convaincante. Cette synthèse met l'accent sur le rôle majeur du canal TREK-1 dans la protection neuronale induite par les AGPI (plus particulièrement les oméga-3) contre deux pathologies neurologiques: I'ischémie cérébrale et l'épilepsie. L'analyse détaillée du rôle des AGPI et de leur cible (le canal TREK-1) dans l'effet protecteur sur les maladies du SNC permet d'envisager de nouveaux traitements pour ces maladies, à la fois dans le cadre de l'urgence médicale mais aussi de la 


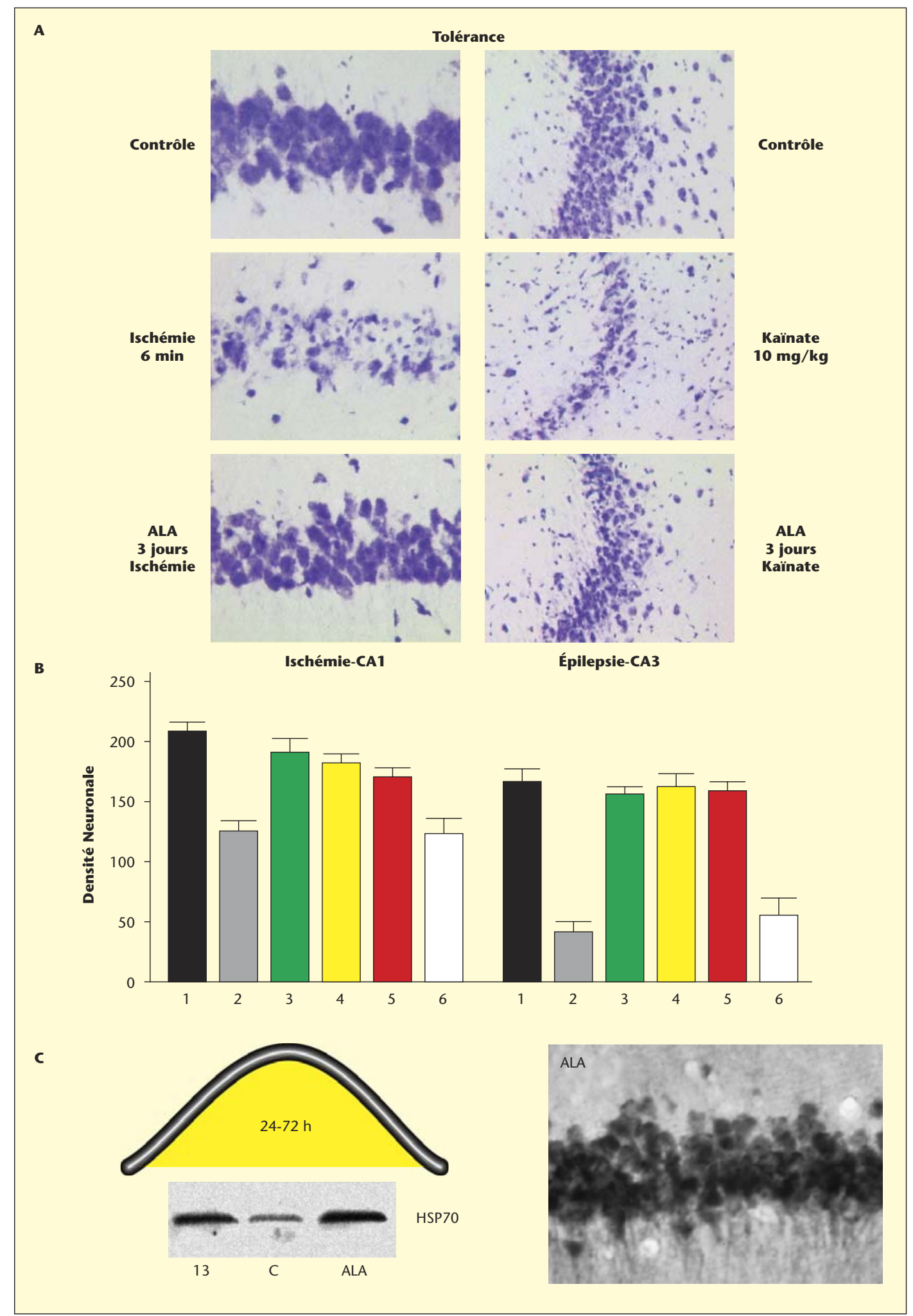

Figure 6. Tolérance ischémique et épileptique induite par un prétraitement aux AGPI. A : Photographies représentatives des régions CA1 (à gauche) et CA3 (à droite) de I'hippocampe 7 jours après les attaques ischémique (durée $6 \mathrm{~min}$ ) ou épileptique (injection $10 \mathrm{mg} / \mathrm{kg} \mathrm{KA}$ ). Effet neuroprotecteur d'une injection intraveineuse de $500 \mathrm{nmol} / \mathrm{kg}$ d'ALA 3 jours avant l'ischémie ou l'épilepsie sévère. B : Histogrammes montrant l'effet protecteur des différents AGPI (prétraitement à 3 jours) sur la densité neuronale

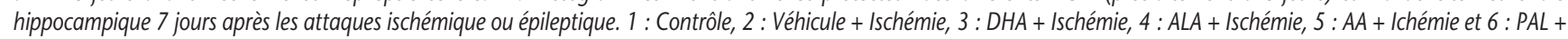
Ischémie. C: Western blot et Immunohistochimie montrant l'induction de la protéine de résistance HSP70 (heat shock protein) dans la fenêtre de protection (24-72 heures) induite par une injection de $500 \mathrm{nmol} / \mathrm{kg}$ ALA (préconditionnement pharmacologique) ou préconditionnement ischémique de 3 min (I3) dans la région CA1 de I'hippocampe. 


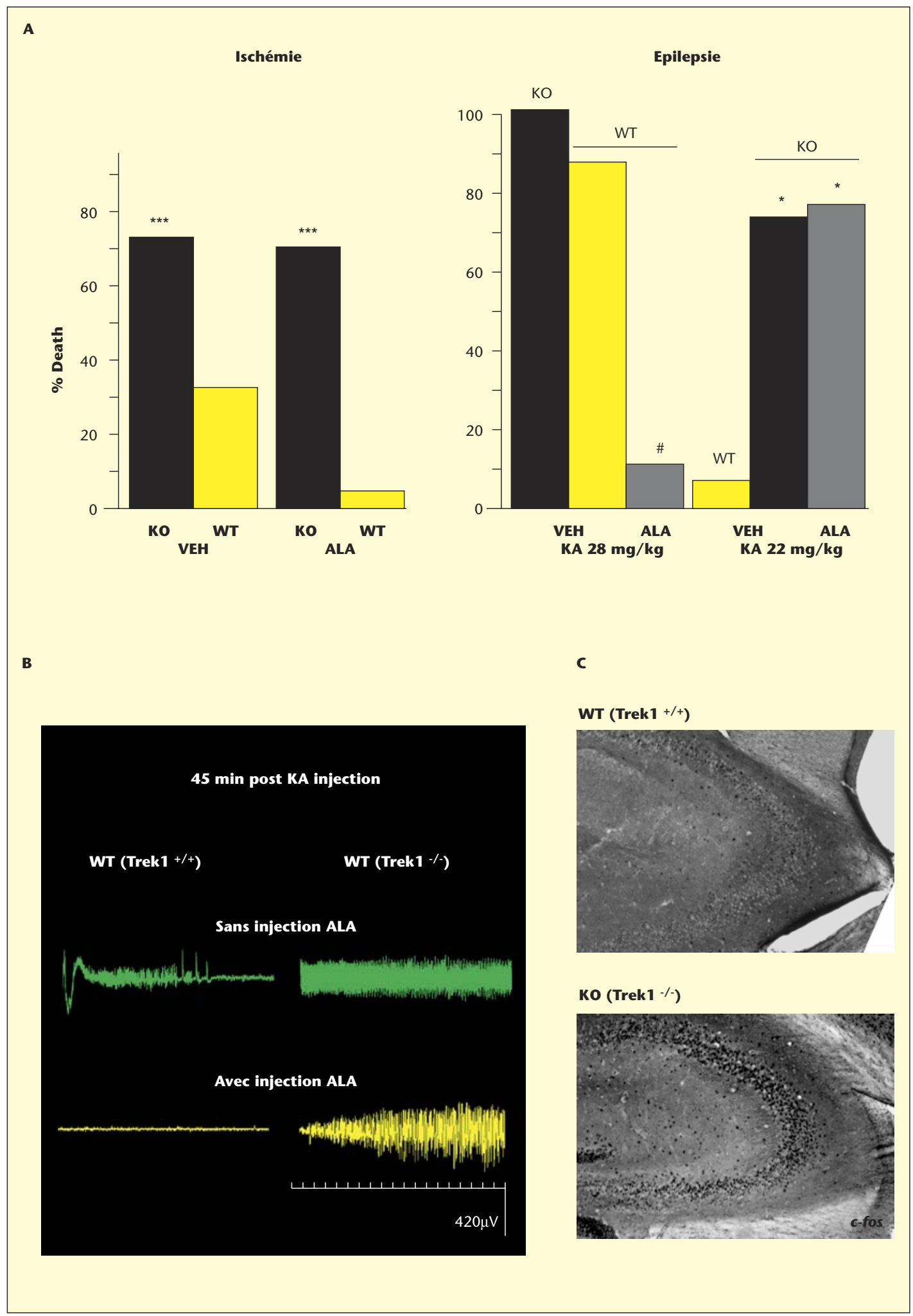

Figure 7. Extrême vulnérabilité des souris TREK1\%à l'ischémie et à l'épilepsie et perte de l'effet neuroprotecteur d'ALA. A. Histogrammes montrant (1) I'augmentation du taux de mortalité des souris $\mathrm{TREK}^{-/-}$suite à une ischémie globale (30 min de clampage des carotides + hypotension) ou à une crise épileptique induite par une injection d'acide kaïnique (22 ou $28 \mathrm{mg} / \mathrm{kg}$ ) ; (2) l'inefficacité d'un traitement à l'ALA (500 nmol/kg) chez les souris TREK1 ${ }^{-1-}$. L'ALA est injecté 30 min avant les attaques ischémique ou épileptique. B : Electroencéphalogrammes enregistrés 45 min après l'injection de $22 \mathrm{mg} / \mathrm{kg}$ de KA montrant I'hyperexcitabilité des souris TREK1-- par rapport aux souris normales et l'absence d'effet antiépileptique de l'ALA (500 nmol/kg). C: Augmentation de l'expression c-fos, un marqueur de l'excitabilité neuronale dans la région CA3 de l'hippocampe, structure vulnérable à l'acide kaïnique. 


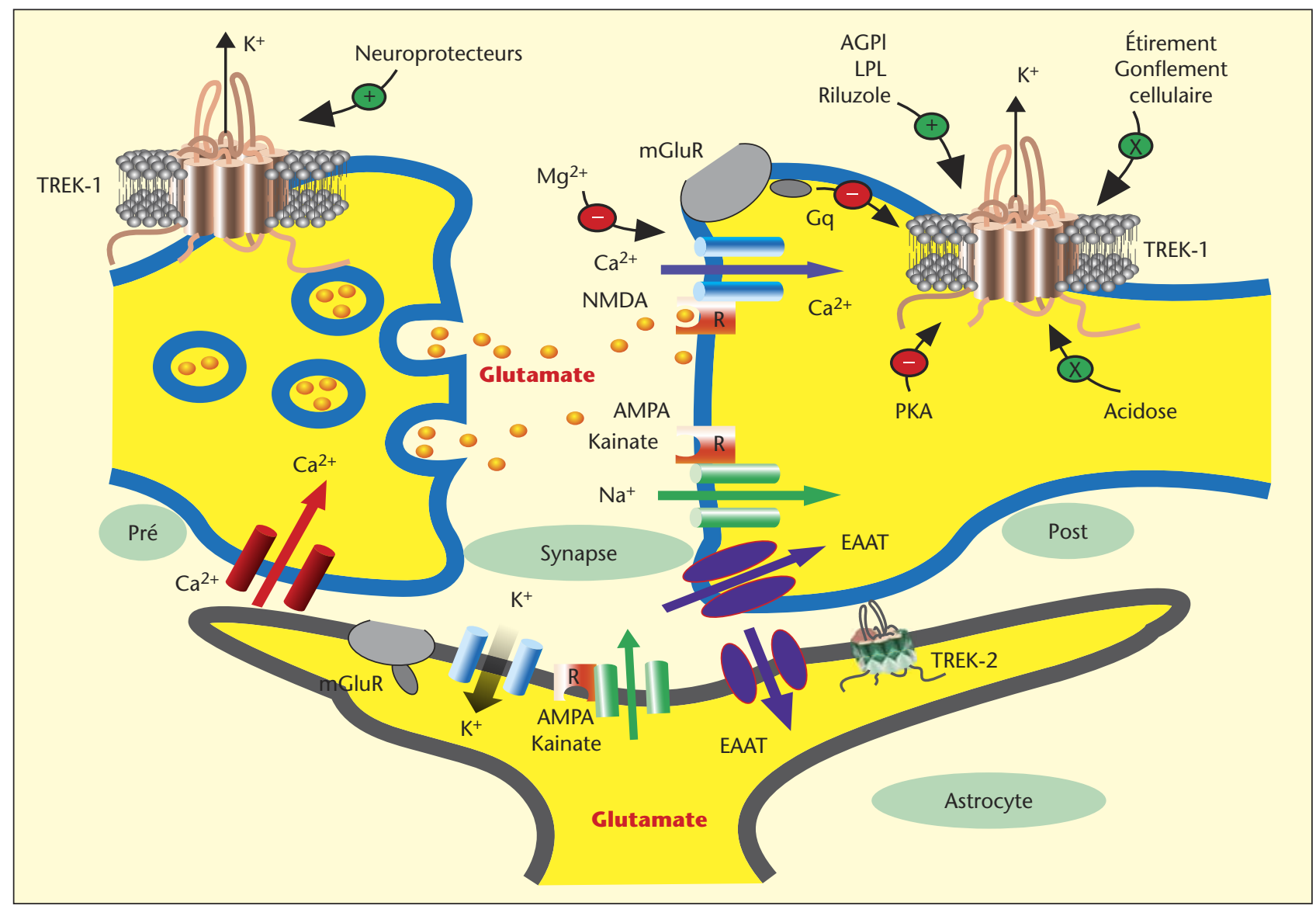

Figure 8. Hypothèse du mécanisme de neuroprotection du canal TREK-1 dans la neurotransmission glutamatergique.

Le canal TREK-1 est présent dans les neurones glutamatergiques et GABAergiques, au niveau pré- et post-synaptique. Les AGPI, les lysophospholipides (LPL) et le riluzole décrits comme étant de puissants neuroprotecteurs contre l'ischémie et l'épilepsie activent fortement le canal TREK-1. L'ouverture du canal TREK-1 au niveau pré-synaptique par les AGPI et les LPL va induire une hyperpolarisation membranaire, qui va réduire la libération du glutamate par fermeture des canaux calciques sensibles au voltage. Au niveau post-synaptique, I'hyperpolarisation membranaire va diminuer l'activation du récepteur NMDA, antagoniser la dépolarisation induite par le récepteur AMPA/kainate et ainsi réduire l'excitotoxicité du glutamate. Par contre, l'activation des récepteurs métabotropiques (mGluR) devrait « down-réguler » le canal TREK-1 par une inhibition directe induite par le diacylglycérol (DAG). Enfin, la présence du canal TREK-2, également activé par les AGPI, les LPL, I'acidose et l'étirement pourrait aussi jouer un rôle dans I'homéostasie potassique au niveau des astrocytes et permettre une meilleure recapture du glutamate par les cellules gliales.

prévention. La découverte de cette nouvelle famille de canaux $\mathrm{K}^{+}$, les canaux K-2P et l'étude de leurs rôles physiologiques et physiopathologiques est d'importance. Quinze membres ont été clonés, chacun avec un profil d'expression particulier et des propriétés fonctionnelles particulières. Certains sont dans les neurones sensoriels, d'autres dans les circuits de la mémoire et de la dépression. Aussi, au-delà de leur rôle dans la vie ou la mort des cellules neuronales, ces canaux sont aussi susceptibles d'avoir un rôle dans le psychisme et d'établir un lien entre neurologie et psychiatrie. Le développement de souris transgéniques pour chacun des membres de cette famille de canaux K-2P n'étant qu'à son début, il n'est pas utopique d'imaginer que les recherches sur ces canaux ouvrent de nouvelles approches thérapeutiques face aux maladies neuropsychiatriques.

Remerciements. Les auteurs tiennent à remercier vivement le Professeur M. Lazdunski de sa confiance et de ses encouragements, ainsi que Mesdames $C$. Widmann et $G$. Jarretou pour leur travail technique remarquable, sans oublier le CNRS et la Fondation Hamel pour leur soutien financier.

\section{RÉFÉRENCES}

1. DELPLANQUE $B$. Acides gras de la famille $n-3:$ alphalinolénique (ALA) d'origine végétale et longues chaînes n-3 (LC-n-3). OCL 2004 ; 11 : 98-102.

2. DUCIMETIERE $P$. Acides gras oméga- 3 et maladies cardiovasculaires : approches épidiémologiques. OCL $2004 ; 11$ : 66-9.

3. DJOUSSEL, HUNT SC, ARNETT DK, PROVINCE MA, ECKFELDT JH, ELLISON RC. Dietary linolenic acid is inversely associated with plasma triacylglycerol: the National Heart, Lung, and Blood Institute Family Heart Study. Am / Clin Nutr 2003; 78 : 1098-102.

4. LEAF A, KANG JX. Prevention of cardiac sudden death by N-3 fatty acids : a review of the evidence. J Intern Med 1996 ; 240 : 5-12.

5. NAIR SS, LEITCH JW, FALCONER J, GARG ML. Prevention of cardiac arrhythmia by dietary $(n-3)$ polyunsaturated fatty acids and their mechanism of action. / Nutr 1997 ; 127 : 383-93.

6. NORDOY A. Dietary fatty acids and coronary heart disease. Lipids $1999 ; 34$ : S19-S22. 
7. SINGH RB, DUBNOV G, NIAZ, ET AL. Effect of an Indo-Mediterranean diet on progression of coronary artery disease in high risk patients (IndoMediterranean Diet Heart Study) : a randomised single-blind trial. Lancet $2002 ; 360: 1455-61$

8. CALDER P. Polyunsaturated fatty acids and inflammation. OCL $2004 ; 11$ : 38-45.

9. BOURRE JM. Omega-3 fatty acids in psychiatry. Med Sci (Paris) $2005 ; 21$ : 216-21.

10. CHALON S. Acides gras polyinsaturés $n-3$, neurotransmission et fonctions cognitives. OCL $2000 ; 7$ : 68-73.

11. LAURITZEN L, HANSEN HS, JORGENSEN MH, MICHAELSEN KF. The essentiality of long chain $\mathrm{n}-3$ fatty acids in relation to development and function of the brain and retina. Prog Lipid Res $2001 ; 40$ : 1-94.

12. STOLL AL, SEVERUS E, FREEMAN MP, ET AL. Omega 3 fatty acids in bipolar disorder: a preliminary double-blind, placebo-controlled trial. Arch Gen Psychiatry $1999 ; 56: 413-6$.

13. HIBBELN JR. Fish consumption and major depression. Lancet $1998 ; 351$ : 1213.

14. BOURRE JM. Roles of unsaturated fatty acids (especially omega-3 fatty acids) in the brain at various ages and during ageing. J Nutr Health Aging 2004 ; 8 : 163-74.

15. BLONDEAU N, WIDMANN C, LAZDUNSKI M, HEURTEAUX C. Polyunsaturated fatty acids induce ischemic and epileptic tolerance. Neuroscience $2001 ; 109: 231-41$.

16. LAURITZEN I, BLONDEAU N, HEURTEAUX C, WIDMANN C, ROMEY G, LAZDUNSKI M. Polyunsaturated fatty acids are potent neuroprotectors. EMBO / $2000 ; 19: 1784-93$

17. LEAF A, KANG JX, XIAO YF, BILLMAN GE, VOSKUYL RA. The antiarrhythmic and anticonvulsant effects of dietary N-3 fatty acids. / Membr Biol 1999 ; $172: 1-11$.

18. LESAGE F, LAZDUNSKI M. Molecular and functional properties of two pore domain potassium channels. Am / Physiol 2000 ; 279 : 793-801.

19. PATEL AJ. Honoré E. Properties and modulation of mammalian $2 \mathrm{P}$ domain K+ channels. Trends Neurosci $2001 ; 24$ : 339-46.

20. HILLE B. Ionic Channels in Excitable Membranes. Ionic Channels in Excitable Membranes. Sunderland, Massachusetts : Sinauer Associates Inc, 1992.

21. SHIEH CC, COGHLAN M, SULLIVAN JP, GOPALAKRISHNAN M. Potassium channels : molecular defects, diseases, and therapeutic opportunities. Pharmacol Rev $2000 ; 52: 557-94$.

22. LESAGE F. Pharmacology of neuronal background potassium channels. Neuropharmacology $2003 ; 44: 1-7$.

23. MAINGRET F, PATEL AJ, LESAGE F, LAZDUNSKI M, HONORÉ E. Lysophospholipids open the two $P$ domain mechano-gated K+ channels TREK-1 and TRAAK. J Biol Chem $2000 ; 275: 10128-33$.

24. LESAGE F, TERRENOIRE C, ROMEY G, LAZDUNSKI M. Human TREK2, a 2P domain mechano-sensitive $\mathrm{K}+$ channel with multiple regulations by polyunsaturated fatty acids, lysophospholipids, and Gs, Gi, and Gq protein-coupled receptors. J Biol Chem 2000 ; 275 : 28398-405.
25. DUPRAT F, LESAGE F, PATEL AJ, FINK M, ROMEY G, LAZDUNSKI M. The neuroprotective agent riluzole activates the two P-domain $\mathrm{K}+$ channels TREK-1 and TRAAK. Mol Pharmacol $2000 ; 57$ : 906-12.

26. FINK M, LESAGE F, DUPRAT F, ET AL. A neuronal two P domain $\mathrm{K}+$ channel stimulated by arachidonic acid and polyunsaturated fatty acid. EMBO / $1998 ; 17$ : 3297-308.

27. PATEL AJ, LAZDUNSKI M, HONORE E. Lipid and mechano-gated $2 P$ domain K(+) channels. Curr Opin Cell Biol $2001 ; 13: 422-8$.

28. LANG-LAZDUNSKI L, BLONDEAU N, JARRETOU G, LAZDUNSKI M, HEURTEAUX C. Linolenic acid prevents neuronal cell death and paraplegia after transient spinal cord ischemia in rats. J Vasc Surg $2003 ; 38$ : 564-75.

29. HOGYES E, NYAKAS C, KILIAAN A, FARKAS T, PENKE B, LUITEN PG. Neuroprotective effect of developmental docosahexaenoic acid supplement against excitotoxic brain damage in infant rats. Neuroscience $2003 ; 119$ : 999-1012.

30. PRATT J, RATAUD J, BARDOT F, ET AL. Neuroprotective actions of riluzole in rodent models of global and focal cerebral ischaemia. Neurosci Lett 1992 ; 140 : 225-30.

31. ETTAICHE M, FILLACIER K, WIDMANN C, HEURTEAUX C, LAZDUNSKI M. Riluzole improves functional recovery after ischemia in the rat retina. Invest Ophthalmol Vis Sci $1999 ; 40: 729-36$.

32. LANG-LAZDUNSKI L, HEURTEAUX C, VAILLANT N, WIDMANN C, LAZDUNSKI M. Riluzole prevents ischemic spinal cord injury caused by aortic crossclamping. / Thorac Cardiovasc Surg $1999 ; 117: 881-9$.

33. MAINGRET F, LAURITZEN I, PATEL AJ. Trek- 1 is a heat-activated background $\mathrm{K}(+)$ channel. EMBO / $2000 ; 19$ : 2483-91.

34. PATEL AJ, HONORE E, LESAGE F, FINK M, ROMEY G, LAZDUNSKI M. Inhalational anesthetics activate two-pore-domain background K+ channels. Nat Neurosci $1999 ; 2$ : 422-6.

35. GRUSS M, BUSHELL T], BRIGHT DP, LIEB WR, MATHIE A, FRANKS NP. TwOpore-domain $\mathrm{K}+$ channels are a novel target for the anesthetic gases xenon, nitrous oxide, and cyclopropane. Mol Pharmacol 2004 ; 65 : 443-52.

36. HEURTEAUX C, GUY N, LAIGLE C, ET AL. TREK-1, a K(+) channel involved in neuroprotection and general anesthesia. EMBO / $2004 ; 23$ : 2684-95.

37. HERVIEU G), CLUDERAY JE, GRAY CW, ET AL. Distribution and expression of TREK-1, a two-pore-domain potassium channel, in the adult rat CNS. Neuroscience $2001 ; 103$ : 899-919.

38. CHEMIN J, GIRARD C, DUPRAT F, LESAGE F, ROMEY G, LAZDUNSKI M. Mechanisms underlying excitatory effects of group I metabotropic glutamate receptors via inhibition of 2P domain $\mathrm{K}+$ channels. EMBO / $2003 ; 22$ : $1-9$.

39. GNATENCO C, HAN J, SNYDER AK, KIM D. Functional expression of TREK-2 $\mathrm{K}+$ channel in cultured rat brain astrocytes. Brain Res 2002 ; 931 : 56-67.

40. VANCASSEL S. Oméga-3 et neurotransmission cérébrale. OCL $2004 ; 11$ : 58-65. 